\title{
The oblique effect in a vernier acuity situation
}

\author{
THOMAS R. CORWIN, ANNE MOSKOWITZ-COOK, and MARC A. GREEN \\ Center for Visual Science, University of Rochester, Rochester, New York 14627
}

\begin{abstract}
Observers viewed either vertical or obliquely oriented vernier targets from either an upright position or with their heads tilted. Vernier acuity was consistently better for retinally vertical than for gravitationally vertical targets, even when presented against a background context of vertical stripes designed to aid veridical perception of gravitational orientation. These results indicate that vernier acuity depends on retinal image orientation rather than on perceived orientation. The high contrast of the vernier lines ensures that their gravitational orientation is clearly perceived. Thus the present results provide a stronger basis for ruling out the effects of perceptual orientation than previous studies involving grating contrast-threshold measurements. Since the vernier targets were presented as brief flashes, it is unlikely that the measured oblique effect is attributable to differences in eye-movement patterns.
\end{abstract}

The normal visual system shows a slight, but significant, preference for horizontal or vertical stimuli over diagonal ones. This phenomenon, known as the oblique effect (Appelle, 1972), has been demonstrated for a number of different stimulus configurations, including vernier targets (Leibowitz, 1955). However, in most previous studies, subjects have viewed the variously oriented stimuli from an upright position. Since, in this situation, the target's perceived orientation and its orientation with respect to the retina are presumably approximately the same, it is impossible to determine whether the visual superiority of a vertical target exists because it is perceptually vertical or because it is vertical with respect to the observer's retina. It is important to remove the ambiguity between these two possibilities, because they imply alternative explanations for the oblique effect. If the effect depends only on retinal image orientation, then it can be explained in terms of differences in orientation sensitivity between neural channels within the visual system alone. On the other hand, if the effect were shown to depend on perceptual orientational coordinates, a more complex explanation would be required-one which took into account variables which affect the perceived orientation of objects, such as visual context, prior adaptational states, and inputs from vestibular, kinesthetic, and proprioceptive sensory systems.

Several recent attempts have been made to dissoci-

This research was carried out at Northeastern University, Boston. It was supported in part by HEW Grant RR 07134 and by PHS Grant EY-01570. A preliminary report of this work was presented to the Eastern Psychological Association, New York, April 1975. The authors thank Warren T. Cobb, Shirley Hartman, Gary Johnson, and Mark Weimersheimer for their assistance. Reprint requests should be addressed to the senior author at Center for Visual Science, Department of Psychology, University of Rochester, Rochester, New York 14627. Anne MoskowitzCook is now at Pennsylvania College of Optometry, 1200 W. Godfrey Ave., Philadelphia, Pennsylvania 19141. ate retinal and gravitational orientational coordinate systems by asking subjects to view stimuli of various orientations with their heads tilted. In this way, a (gravitationally) vertical target may be made retinally oblique and an oblique target may be made retinally vertical. Four experimental conditions may be used: (1) a gravitationally vertical target presented to an observer whose head is upright, (2) a tilted target presented to an observer whose head is upright, (3) a vertical target presented to an observer whose head is tilted, and (4) a tilted target presented to an observer whose head is tilted to the same extent as the target. If it is assumed that the observer accurately perceives the gravitational orientation of the targets (and neglecting the influence of countertorsion of the observer's eyes when his head is tilted), we may conclude that (a) Conditions 1 and 4 differ in perceived orientation but not in retinal orientation, and (b) Conditions 2 and 3 differ in perceived orientation but not in retinal orientation. Conditions 1 and 4 are both retinally upright, whereas Conditions 2 and 3 are both retinally oblique, although the two pairs differ in perceptual orientation. If, therefore, no significant differences in visual performance is found between Conditions 1 and 4 or between Conditions 2 and 3, it may be concluded that perceived orientation has no significant influence on the visual task. Furthermore, if performance is significantly better for Conditions 1 and 4 than for Conditions 2 and 3 , this may be attributed to the differences in retinal orientation, demonstrating that the oblique effect depends on retinal orientation.

Both these conclusions depend on the assumption that the orientation of the targets in the four conditions is perceived veridically, that is, that the target's perceived orientation is at least approximately equivalent to its gravitational orientation. If, for example, experimental conditions are such that perceptual compensation for head tilt is incomplete or absent, 
the effective dissociation between retinal and perceptual orientational coordinates would be reduced, or even eliminated. Clearly, the possibility of incomplete compensation tends to vitiate conclusions concerning the relative visual effects of changes in perceptual and retinal orientations. Compensation for head tilt is particularly ineffective when illumination is dim, and probably also when stimuli are at very low contrast levels. For example, Ross (1974) states: "The most common source of conflict (between cues to the vertical) is between the visual and the vestibular-kinaesthetic vertical. A potential conflict of this sort arises when the eye is tilted, due to head or body tilt, so that the retinal image of a vertical object is no longer 'upright' on the retina. There need be no conflict of course, provided the brain has adequate vestibular-kinaesthetic information about the orientation of the retina, and can use the information to correct the orientation that the retinal image indicates. In broad daylight the compensating system works well, and the world appears upright even when the head is tilted. In the dark, this is no longer the case, and a glowing bar appears tilted when the observer is tilted"' (p. 105).

In three recent psychophysical studies (Banks \& Stolarz, 1975; Findlay \& Parker, 1972; Lennie, 1974), the head-tilt technique was used to measure contrast thresholds for dim gratings. All three studies found that the oblique effect depends on retinal orientation and is relatively independent of changes in gravitational orientation. However, because visual performance in these studies was measured with the gratings at just detectable contrast levels and at relatively low levels of total luminance, it is likely that perceptual compensation for head tilt was incomplete. Thus, the studies may have failed to achieve optimal dissociation of retinal and perceptual coordinate systems.

The purpose of the present study was to optimize dissociation between retinal and perceptual orientations, by setting up conditions favorable to effective perceptual compensation for head tilt. A vernier target is ideal for this purpose because it provides a reliable measure of visual acuity, while the orientation of the target lines is always seen clearly and precisely.

There have been few studies in which the head-tilt paradigm has been applied to suprathreshold targets, but the conclusions reached differ, depending on the type of response measured used.

For example, Attneave and Olsen (1965) found longer reaction times for (gravitationally) oblique targets than for vertical targets, regardless of whether the observer's head was tilted or upright. This implies that reaction times depend on perceived rather than on retinal orientation. Kitterle (1973), on the other hand, found that rectangular simultaneous-contrast targets yielded higher contrast ratings when retinally vertical than when retinally oblique, despite differences in their perceived orientation. This implies that apparent contrast varies with retinal rather than perceived orientation.

Frost and Kaminer (1975) recorded visually evoked cortical responses for vertical and oblique gratings during head. tilt. Cortical responses to retinally vertical targets were larger than to gravitationally vertical ones, implying that evoked potential responses vary with retinal rather than with perceived orientations.

The present study extends the head-tilt paradigm to a vernier acuity situation. A dark vernier acuity target, seen against a relatively intense circular background, was used. The targets were presented as brief flashes to minimize the effects of eye movements. In one condition, room illumination was increased and a background context was provided to further support the observer's veridical perception of gravitational orientation. In all cases, acuity scores for retinally vertical, perceptually oblique targets were higher than those for retinally oblique, perceptually vertical targets. This demonstrates that vernier acuity varies with retinal orientation, and is relatively unaffected by changes in perceived orientation.

\section{METHOD}

\section{Stimuli}

The vernier acuity target was made from two pieces of $1 / 16 \mathrm{th}$ in. $(1.59-\mathrm{mm})$ matte black Lettraset tape. One piece was mounted on a fixed diffusing plate of translucent plastic, and the other was mounted on a thin glass plate which could be moved back and forth over the fixed plate with a micrometer movement. Figure $1 \mathrm{~b}$ shows the target as it appeared to the observers (the 35-deg inclination of the target is slightly exaggerated in the figure). At the $2.08-\mathrm{m}$ viewing distance used, the vernier lines were $2.75 \mathrm{sec}$ arc wide and 2.27 deg long.

\section{Apparatus}

The target device rested on a horizontal level platform, transilluminated from below by light from a Kodak Carousel projector. The target was illuminated for each trial for $10 \mathrm{msec}$ under the control of a 26-mm-diam shutter (Uniblitz Model 225; nominal rise-time: $5 \mathrm{msec}$ ) driven by a preset digital counter-timer (Iconix Model 6255). An image of the vernier target was reflected into the observer's frontal plane by a mirror located above the platform (Figure 1a). Subjects viewed this image through a circular aperture (2.27-deg-diam subtense) cut from a large opaque baffling screen. Viewing distance was $2.08 \mathrm{~m}$. To facilitate fixation, the "break" in the vernier target was aligned to coincide with the center of the circular aperture. The angle of the vernier target with respect to the gravitational vertical was established by careful positioning of the target device on the horizontal platform. Two orientations were used: 0 deg (gravitationally vertical) and $35 \mathrm{deg}$ (measured counterclockwise from the horizontal). The accuracy of these orientations was checked by projecting an image of the target on a vertical screen temporarily located at the observer's viewing position, and measuring the image's orientation with a plumb-bob and protractor.

Subjects viewed the targets with the right eye, either from an upright position (head position maintained with a chinrest and forehead brace) or while supported in a tilted position by a specially designed tilted chair. The chair maintained the observer's head and body at an angle of $35 \mathrm{deg}$ from the horizontal. [At 


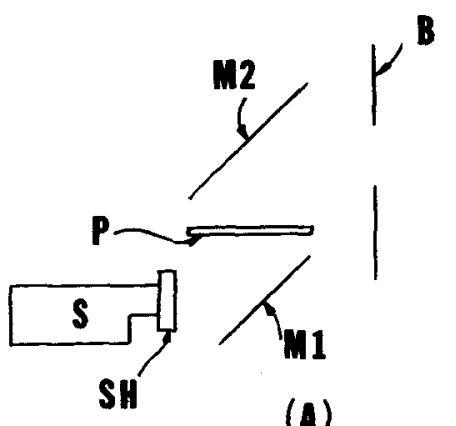

(A)

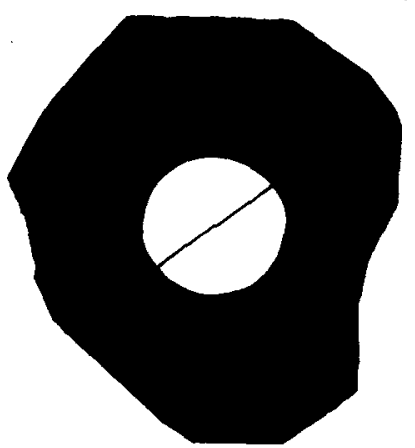

(B)

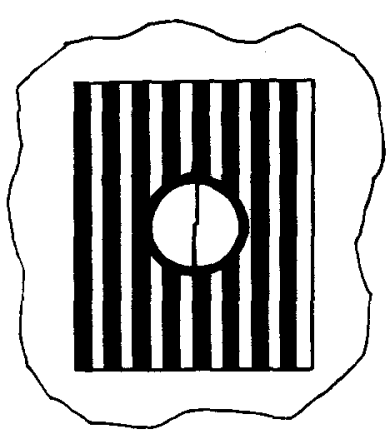

(C)
Figure 1. (a) Side view of apparatus. Vernier target rested on horizontal platform P. Target was transilluminated by light from source $S$ reflected by mirror $M_{1}$. Image of target was reflected toward observer by mirror $M_{2}$ through circular stop in baffle $B$. (Drawing not to scale.) (b) Target shown in tilted orientation from observer's point of view under dark room condition. Actual tilt angle was 35 deg from horizontal. (c) Target shown in upright orientation from observer's point of view under context cues condition.

this inclination, it is estimated that the observer's eyes counterrotate by about $3.8 \mathrm{deg}$ (see Moskowitz, 1974, p. 197-199); the effect of this small angle was ignored in setting target orientation.] Head position was maintained by a tilted chinrest and forehead brace. The subject's left eye was covered with an eyepatch. Subjects initiated each trial when ready by pressing a telegraph key to activate the shutter driver.

Acuity measurements were made under two conditions. In the first condition, the circular vernier display was seen in an essentially dark room. Stray light from the light source provided diffuse illumination of the target area to allow accurate fixation. During the 10-msec target exposure, the vernier target appeared within a circular (2.27-deg-diam subtense) homogeneous field of 660 -fL.

In the second experimental condition, additional context cues to gravitational coordinates were provided by illuminating the experimental room. The light source was a ceiling fixture containing two adjacent 6-ft fluorescent tubes mounted parallel to the observer's line of sight. To provide further context cues to the vertical, the vernier target was surrounded by a large $(22 \times 27 \mathrm{deg}$ subtense) rectangular baffle containing an array of vertical squarewave stripes (Figure 1c). The vernier target was projected through a circular hole (1.7-deg-diam subtense) cut from the center of the baffle. There were 13.5 black/white cycles within the field of view; the contrast of the stripes was 0.78 , and their spatial period was $14.3 \mathrm{~min}$ of arc (4.2 cycles per degree). The mean luminance of the array of stripes was $19.0 \mathrm{~cd} / \mathrm{m}^{2}$ and the ambient luminance of regions surrounding the array was $21.9 \mathrm{~cd} / \mathrm{m}^{2}$. All luminance calibrations were carried out from the observer's viewing position with a Spectra brightness spotmeter (Model 1505 UB).
In both experimental conditions, the vernier target was exposed for $10 \mathrm{msec}$ in a trial. In the dark room condition, the shutter was mounted directly in front of the light source, and in the context cues condition, the shutter was mounted behind the cardboard baffle on which the array of stripes was located. This change caused a difference in dimensions of the circular region in which the vernier target appeared (cf. Figure $2 b$ and 2c). No other changes in the apparatus were made between the two conditions.

\section{Subjects}

Four undergraduate students, three male and one female, served as volunteer subjects. All were well-practiced psychophysical observers. Three of them required no visual correction, either spherical or cylindrical; the fourth wore corrective spectacles during the sessions. A number of other people, including the authors, served as observers in preliminary pilot work in which a variety of combinations of background luminance and target duration were investigated. The pilot data agree well with the results of the undergraduate observers reported here.

\section{Procedure}

Vernier acuity performance was measured using an alternating method of limits with a three-alternative response. The lower linesegment of the vernier target was moved by the experimenter between trials, in steps of $0.2 \mathrm{~mm}(0.35 \mathrm{sec}$ arc; or approximately 1 receptor diameter). Each acuity measurement consisted of 10 series of displacements, five from either direction. The initial displacement of each series was randomly varied to preclude step counting.

Cumulative response curves were plotted as a function of linesegment displacement in millimeters. Data was quantified by obtaining a least squares linear fit to each psychometric function and using the slopes of these best fitting lines to calculate the displacement producing a $50 \%$ probability of correct detection. The reciprocal of these just-noticeable displacements, expressed in minutes of arc, constituted our measure of vernier acuity. Each acuity score is based on approximately 50 individual judgments.

Acuity was measured under four conditions of observer orientation and target orientation: observer upright/target upright, observer upright/target tilted, observer tilted/target upright, observer tilted/target tilted. Within a given session, measurements were made for both vertical and tilted targets; the observer's orientation was not changed within a session. The order in which target orientations were presented within a session was randomized.

\section{RESULTS AND DISCUSSION}

Results for both experimental conditions are plotted in Figure 2. The four combinations of head orientation and target orientation are indicated on the abscissa (target orientation slightly exaggerated). The two points at the ends of the axis represent conditions in which the target is oriented vertically with respect to the observer's retina, and the two middle points represent conditions in which the target is oblique with respect to the observer's retina. Vernier acuity, plotted on the ordinate, is expressed as the reciprocal of the target displacement (in minutes of arc) correctly detected by the observer with a probability of 0.5 . Thus, increasing acuity is plotted upward.

Comparing the two subject-upright conditions (the two leftmost abscissa points of either graph in Figure 2), it can be seen that acuity scores are con- 


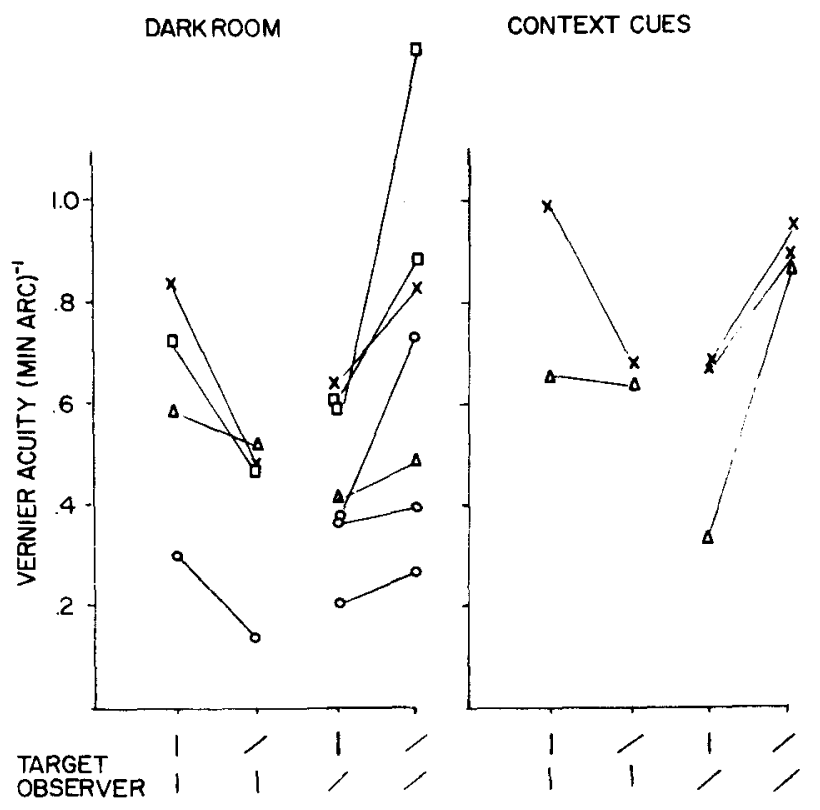

Figure 2. Vernier acuity scores as a function of orientation of target and of observer's head for dark room condition (left) and context cues condition (right). Each point is based on approximately 50 individual judgments; each observer is represented by a different geometric symbol. Lines connect acuity scores measured within a single session for each observer.

sistently lower for the obliquely oriented target than for the upright target (sign test, $\mathrm{p}<.05$, two-tailed). This result confirms the existence of an oblique effect for vernier acuity, previously demonstrated by Leibowitz (1955). The present results extend his finding by demonstrating that the effect is present under conditions of very brief target presentations as well as for continuous viewing. This form of presentation minimizes the influence of eye movements on performance, and so tends to rule out the possibility that the oblique effect is attributable to differences in eye-movement patterns (see also Higgins \& Stultz, 1950; Nachmias, 1960).

Comparing pairs of vernier acuity scores obtained within single sessions yields a measure of the relative decrement in acuity produced by a change in retinal orientation from vertical to $35^{\circ}$. Averaged over all 16 pairs of data points included in Figure 2, the acuity decrement approximates $30 \%$. This compares to a decrement of $20 \%$ found by Leibowitz for a 45-deg change in target orientation.

It should be noted that the present results, as well as Leibowitz' earlier finding, contradict the implication of a model of vernier acuity recently proposed by Findlay (1973). Findlay hypothesized that the "kink" in a vernier target is detected by a linedetecting "channel" whose preferred axis is oriented obliquely with respect to the vernier target lines. When he made acuity measurements with the vernier target superimposed on backgrounds of variously oriented suprathreshold gratings, he found that gratings which maximally disrupted acuity had orientations of about $20 \mathrm{deg}$ in either direction with respect to the vertical vernier lines. This result implies optimal acuity for a vernier target oriented $20 \mathrm{deg}$ away from the vertical or horizontal, since, when the vernier lines are oriented at these angles, the "kink" is being detected by neural units, the preferred orientations of which are vertical or horizontal. Specifically, Findlay's results imply that vernier acuity will be at a relative minimum for target orientations of 0,45 , and $90 \mathrm{deg}$ with respect to the vertical, with relative maxima occurring at 20 and $70 \mathrm{deg}$. In the present study, vernier acuity was measured at orientations of 0 and $55 \mathrm{deg}$ with respect to the vertical (20 and $15 \mathrm{deg}$ away from the orientations predicted to yield maximal sensitivity, respectively). For these orientations, Findlay's model predicts little difference in acuity, with that difference tending toward superior acuity for the retinally oblique target. Neither the present results nor those of Leibowitz confirm the prediction. A resolution of the issue awaits the careful measurement of vernier acuity over a wide range of target orientations.

A comparison of corresponding acuity scores for the two observers who participated in both the dark room and the context cues conditions shows that acuity scores are improved for the context cues condition relative to the dark room condition in 9 out of 10 cases (sign test, $p<.05$, two-tailed). This fact is consistent with Craik's (1938) finding that visual acuity is maximized when the observer's eye is adapted to a level of illumination approximating that of the target. It is possible (but unlikely) that the presence of the array of context stripes themselves contributed to the improvement in acuity. This possibility remains to be tested.

There were no systematic differences in acuity between the two conditions in which the target was retinally oblique (i.e., observer upright/target tilted and observer tilted/target upright). Nor were any systematic differences in acuity scores found between the two conditions in which the target was retinally vertical (i.e., observer upright/target upright and observer tilted/target tilted). Real differences between these pairs of experimental conditions may have been obscured by between-session variability, however, since neither of the pairs was tested within a single session.

The most important result is the difference in acuity scores for the two observer-tilted conditions (the two rightmost points on the abscissa of Figure 2). In all 10 cases measured, with or without augmented cues to gravitational coordinates, retinally oblique targets yielded lower acuity scores than did retinally vertical targets (sign test, $p<.05$, twotailed). These results provide clear evidence that vernier acuity is not appreciably affected by a target's 
perceived orientation in space, but instead depends predominately upon its orientation on the observer's retina.

\section{REFERENCES}

APPElle, S. Perception and discrimination as a function of stimulus orientation: The "oblique effect" in man and animals. Psychological Bulletin, 1972, 78, 266-278.

Attneave, F., \& Olson, R. K. Discriminability of stimuli varying in physical and retinal orientation. Journal of Experimental Psychology, 1967, 74, 149-157.

Banks, M. S., \& Stolarz, S. J. The effect of head tilt on meridional differences in acuity: Implications for orientation constancy. Perception \& Psychophysics, 1975, 17, 17-22.

CrAIK, K. J. W. The effect of adaptation upon visual acuity. British Journal of Psychology, 1938, 29, 252-266.

FinDLAY, J. M. Feature detectors and vernier acuity. Nature, 1973, 241, 135-137.

Findlay, J. M., \& Parker, D. M. An investigation of visual orientation constancy using orientation-specific properties of acuity and adaptation. Perception, 1972, 1, 305-313.
Frost, B. J., \& Kaminer, J. J. The orientation anisotropy and orientation constancy: A visual evoked potential study. Perception, 1975, 4, 51-58.

Hrgains, G. C., \& Stultz, K. Variation of visual acuity with various test-object orientations and viewing conditions. Journal of the Optical Society of America, 1950, 40, 135-137.

KutTerLe, F. The possible locus of lightness contrast. Perception \& Psychophysics, 1973, 14, 585-589.

LEIBOWITZ, H. Some factors influencing the variability of vernier judgments. American Journal of Psychology, 1955, 68, 266-273.

LENNIE, P. Head orientation and meridional variations in acuity. Vision Research, 1974, 14, 107-111.

Moskowitz, A. F. The interaction of head position and stimulus orientation in human vision. Unpublished $\mathrm{PhD}$ thesis, Northeastern University, Boston, 1974.

Nachmias, J. Meridional variations in visual acuity and eye movements during fixation. Journal of the Optical Society of A merica, 1960, 50, 569-571.

Ross, H. B. Behavior and perception in strange environments. London: Allen \& Unwin, 1974.

(Received for publication January 17, 1977; revision accepted March $17,1977$. 